\title{
Ethiktransfer und Literatur
}

Erhard Taverna

${ }^{*}$ www.dialog-ethik.ch

www.fhnw.ch/sozialearbeit

erhard.taverna[at]saez.ch
1. Ist Ethik ansteckend, kann man sie übertragen wie eine Bluttransfusion? Und wer spendet da was und wem? Doch zuerst einige propädeutische Klärungen: Ethik als Philosophie versteht sich als eine Wissenschaft, die sich mit den Grundsätzen eines guten, richtigen und gerechten Handelns befasst. Ethik ist eine Theorie der moralischen Praxis von Individuen (Individualethik), Gruppen (Sozialethik) und Institutionen (z.B. Bildungs-, Gesundheits-, Wirtschaftswesen). Ziel ist die praktische Beratung im Rahmen eines Austauschprozesses zwischen den Experten und Akteuren der angesprochenen Bereiche (Bereichsethik). Der Vorgang gleicht einer Aufklärung, die eine ethische Urteilsbildung ermöglichen soll. Für die organisatorische Umsetzung von Ethik in unterschiedlichen Handlungsfeldern steht der unschöne Begriff «Ethiktransfer», in Analogie zum Begriff des «Technologietransfers», der die Nutzung und Verbreitung von Forschungs- und Technologieergebnissen beinhaltet. Mit Transfer ist nicht die einseitige Spende oder Anweisung, sondern der Austausch zwischen A und B gemeint.

2. Wo Menschen sich nicht mehr dem Absolutheitsanspruch einer Religion unterziehen wollen, ist die Nachfrage nach Ethik gross. Aber auch dort, wo nur partikulare Interessen miteinander konkurrie-
Staat, der, im Rahmen seiner Sparpolitik, zunehmend die Verantwortung für das soziale und gesundheitliche Risiko auf die Bürger und Bürgerinnen abschiebt. Die Medizinethik spricht von einer Responsibilisierung des Patienten, dem im Namen von Autonomie und Selbstverantwortung Dienste entzogen und dafür Pflichten aufgebürdet werden.

3. Alle Bereichsethiken arbeiten theoretisch oder für Schulungen mit Gedankenexperimenten und Fallbeispielen. Für die Urteilsbildung müssen erst einmal die Probleme bekannt, die Alternativen abgewogen, die Normen geprüft und die Folgen analysiert sein. Im Idealfall finden sich die abweichenden Meinungen in einem mehrheitsfähigen Entscheid, der für alle Beteiligten verbindlich umgesetzt wird. Die theoretische Ethikdiskussion erschafft sich oft eine eigene Realität, abgehoben vom real existierenden Alltag. Darum Ethiktransfer, er soll den Kontakt mit dem Bodenpersonal wieder herstellen.

4. In Zusammenarbeit mit der Fachhochschule Nordwestschweiz organisiert das Institut «Dialog Ethik» einen Abschluss Master of Advanced Studies (MAS) «Ethische Entscheidungsfindung in Organisation und Gesellschaft»*. Dazu gehört auch der Wahlpflicht-Zertifikatskurs «Ethik und Literatur im Dialog», der als einziges Modul besucht werden

\section{«Wo Menschen sich nicht mehr dem Absolutheitsanspruch einer Religion unterziehen wollen, ist die Nachfrage nach Ethik gross.»}

ren, besteht Bedarf nach einer neutralen Instanz. An die Ethik werden heute grenzenlose Ansprüche gestellt. Sie soll eine Art Mediation zwischen den vordergründig unvereinbaren Positionen verschiedener Gesellschaftsgruppen in Gang setzen, den Medizinstudenten zu einem kritischen Orientierungswissen verhelfen, für Politiker Technologiefolgen abschätzen und für die Naturwissenschaft eine Frühwarnfunktion erfüllen. Inzwischen gibt es unzählige Gremien auf allen Stufen öffentlicher und privater Unternehmen, von denen allgemeingültige Verhaltensregeln für Konfliktfälle erwartet werden. Bewährt haben sich diese Einrichtungen als institutionalisierte Entwicklungshilfe für Problemlösungen in den Grenzzonen der Bioforschung, der modernen Medizin und einiger Naturwissenschaften. Enttäuschend sind die Resultate in den Domänen der Wirtschaft, allen voran des Finanzsystems. Ethik findet nicht im luftleeren Raum statt. Sie kann ihrerseits instrumentalisiert werden, zum Beispiel durch einen kann. Die Teilnehmer in Oerlikon sind eine leitende Ärztin, Heimleiterinnen, ein Spitalpfarrer und Pflegekader, unter Leitung des belgischen Theologen und Philosophen Jean-Pierre Wils. Ethische Grundfragen werden während zweitägiger Seminare mittels literarischer Texte erarbeitet. Es geht um existentielle Herausforderungen wie: Sterben und Tod, Verantwortung, Schuld und Sühne, das Leiden der Kreatur und den Widerstreit der Gefühle.

Schriftsteller strukturieren das Chaos. Die Texte werden als Versuchsanordnungen, mit unterschiedlichen Protagonisten, Fragen und Analysen gelesen. Die Erzählungen verdichten die Erfahrungen und Entwürfe ihrer Figuren, vergleichbar einem Laborexperiment, das die unterschiedlichsten Lebensverläufe und Abhängigkeiten einer geplanten Geschichte unterordnet. Die Autoren schaffen Distanz und Nähe, sie verlangsamen oder beschleunigen, sie überschreiten Zeit und Raum, sie schaffen das Arsenal der Metaphern. Die Vielfalt der Stile und Pers- 
pektiven verhindert eine akademische Verschulung. Ein guter Text fordert den Leser heraus und hält ihm einen Spiegel vor, indem dieser sich in den Figuren wiederfindet, in ein fremdes Schicksal hineingezogen, vielleicht sogar verändert wird. Wie bei allen ethischen Fragen muss er letztlich selber entscheiden, auf welche Seite er sich schlagen will.

Die Kursteilnehmer lernen allgemeine Prinzipien der Philosophie kennen und erweitern über die vorbereitete Buchlektüre ihren Horizont. Ohne Sprache gibt es keine Selbstreflexion. Vielleicht kann man sogar behaupten, dass ohne Weltliteratur keine Praxis der Ethik möglich ist. Nur die Antennen dieser Schriftsteller und Schriftstellerinnen übersetzen das tägliche Hintergrundrauschen in eine verständliche Botschaft. In Zeiten immer weiter zunehmender Differenzierung des Fachwissens erlaubt die Lektüre einen Blick über den Tellerrand. Sie leistet einen lebenslänglichen Wissens- und Empathietransfer, der den Verstand und die Sinne wachhält. Fiktive Geschöpfe und Handlungen ermöglichen Identifikationen mit fremden Menschen, damit auf den Spielfeldern der Sprache die Voraussetzungen für ein $\mathrm{Zu}-$ sammenleben gedeihen. Texte vermitteln Konfliktstrategien meist besser als Bildmedien. Wo es um zwischenmenschliche und integrative Leistungen geht, sind sie überlegen, weil sie das Unaussprechliche formulieren. «Ich glaube, man sollte überhaupt nur Bücher lesen, die einen beissen und stechen», schrieb Franz Kafka in einem Brief, und etwas weiter: «... ein Buch muss die Axt sein für das gefrorene Meer in uns». Genau darum geht es in diesem Kurs. Die Lektüre verführt zu weiterer Lektüre, damit das Gelesene mithilft, Verhärtetes aufzubrechen, Erstarrtes zu bewegen, Geformtes zu verflüssigen, einen Dialog mit sich und anderen in Gang zu setzen. 